\title{
A Review of Autorefractors
}

\author{
I. C. J. WOOD
}

Manchester

\begin{abstract}
Summary
The overall performance of autorefractors in this study as defined by reliability, validity and rejection rate statistics, is similar to other hospital based studies but lower than in an optometric clinic., ${ }^{4,1,5,6}$, The performance of individual autorefractors varies according to the interaction between the underlying optical/detector mechanism and the media and pathological changes of a given eye. The autorefraction data proves a useful complement to the refractive data gained by conventional techniques, notwithstanding the poorer performance of the autorefractors in the presence of senescent pathology.
\end{abstract}

It is almost half a century since Collins ${ }^{7}$ reported the design and construction of the first objective infrared optometer. However, the first fully automated infrared optometer, the Ophthalmotron, did not appear until 1970 . Recent years have seen a rapid proliferation of commercially available instruments, each offering the same basic specification for measuring refractive errors: $+/-15$ dioptre sphere, cylinder powers up to 7 dioptres in 1 degree axis settings.

Recent studies ${ }^{1,3}$ and some review articles ${ }^{8,9}$ of autorefractors indicate that reliable and valid preliminary refraction measurements can be obtained from these instruments. Therefore a comparison study of five currently available autorefractors the Canon Ref10, Dioptron V (CooperVision), the Humphrey 510 autorefractor, the Nidek 3300 autorefractor and Nikon 2000 autorefractor was undertaken at Manchester Royal Eye Hospital to assess the accuracy and overall performance of these instruments.

\section{Methods}

The reliability and validity of the measurements of each autorefractor was compared with retinoscopy 10,5 or conventional refraction techniques. $4,11,1,6,12,13,3$

Reliability is the consistency with which measurements are made, i.e. their repeatability. The reliability statistics were therefore generated from repeated autorefraction measurements preferably made on separate occasions.

Validity on the other hand, is the degree to which tests measure what they say the measure. It has been pointed out by many authors that conventional refraction techniques are prone to bias and error. ${ }^{14,2,12}$ However they are accepted as the norm and therefore it is natural that the conventional refraction measurements form the criteria against which the autorefraction measurements should be validated. The statistics were obtained by comparing the autorefraction measurements with the results of a blind conventional refraction carried out by Dr. J. Storey and his staff of optometrists at Manchester Royal Eye Hospital. The visual acuities obtained with both autorefraction and conventional refraction were recorded. The statistical analysis was generated by a SPSS Package on the UMRCC CDC 7600 mainframe computer.

\section{Results}

Distribution of Age and Refractive Error

It is clear from Figure 1 that over half the three

Correspondence to: I. C. J. Wood PhD, MSc, FBCO, Lecturer in Ophthalmic Optics, University of Manchester Institute of Science \& Technology P.O. Box 88 Manchester M60 1QD.

Presented at the Annual Meeting of the Ophthalmological Society of the United Kingdom, April 1986. 


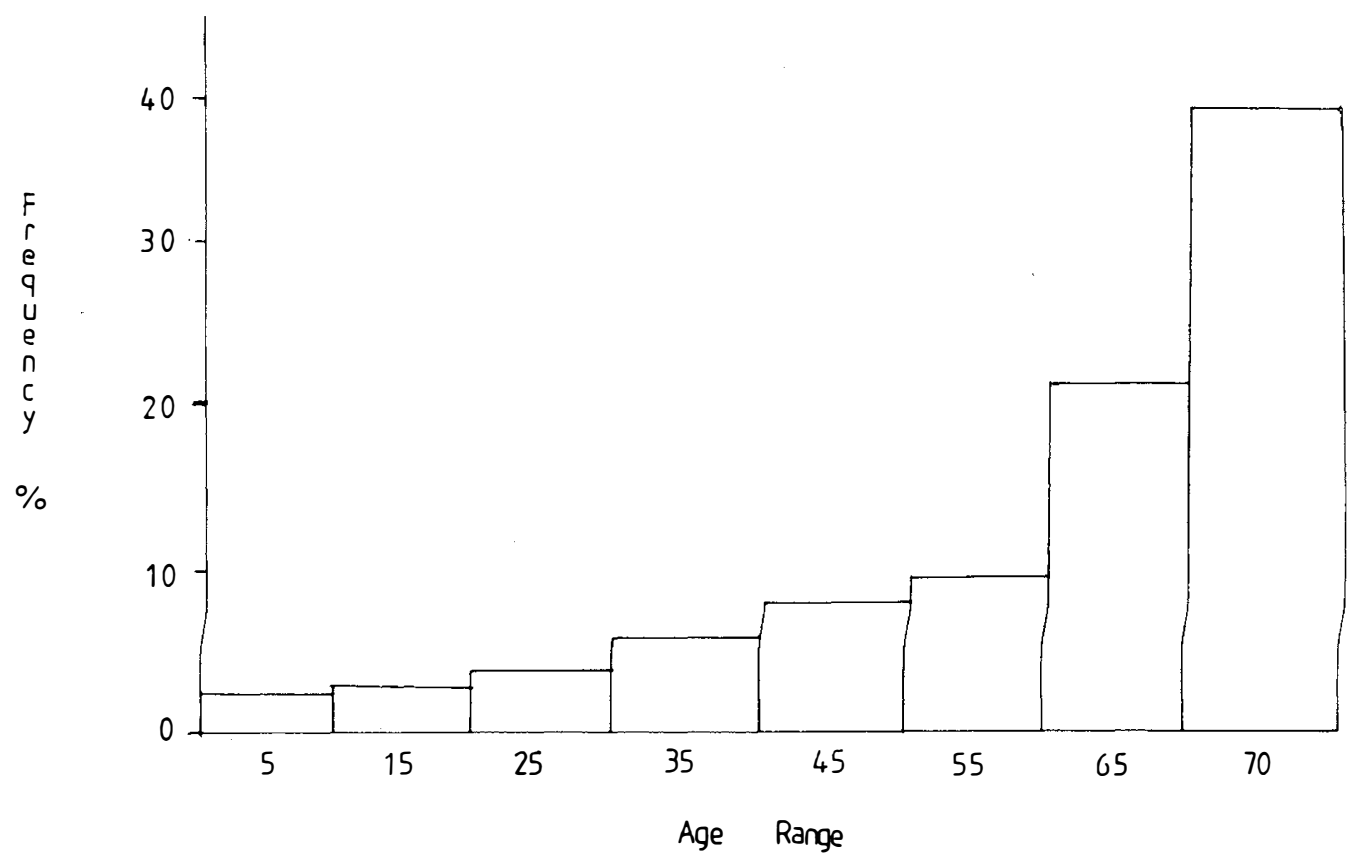

Fig. 1. Distribution of age of the patients in the present study.

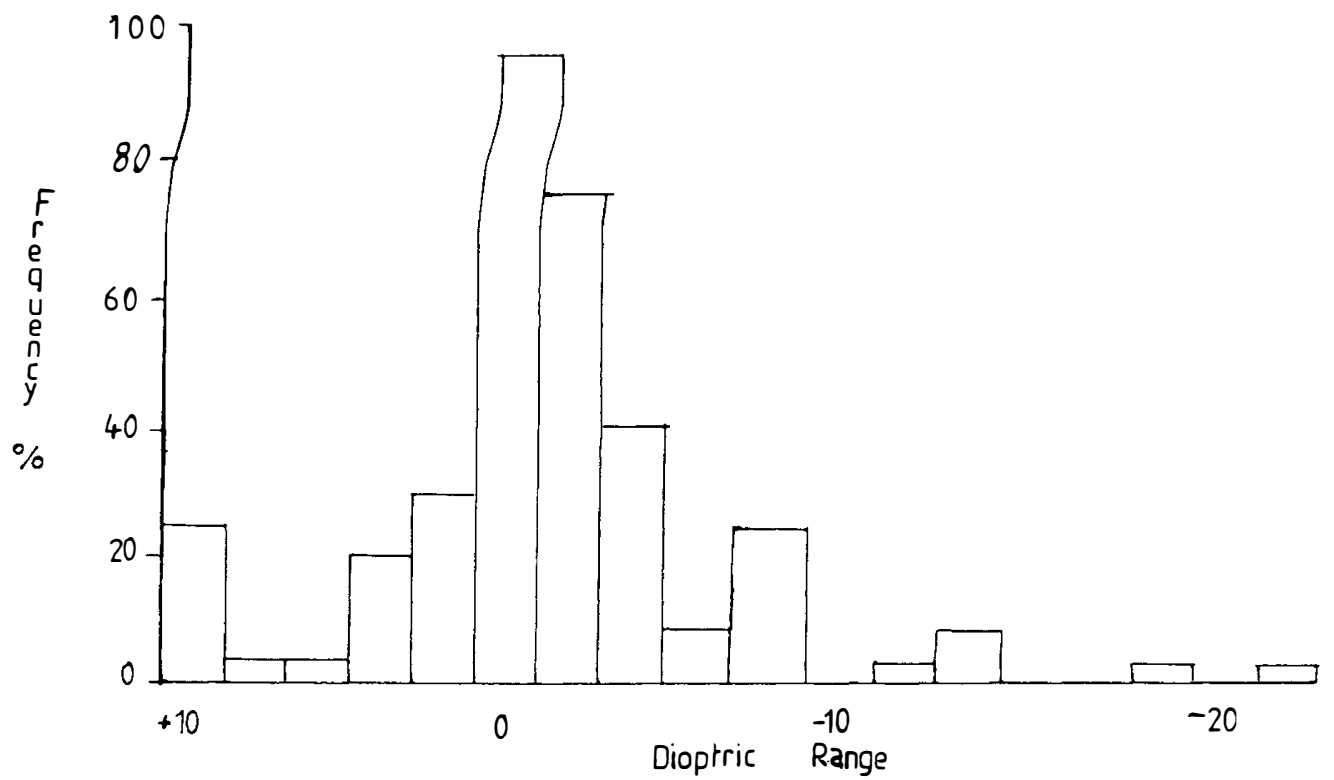

Fig. 2. Distribution of Refractive Error (Spherical Equivalent) of the patients in the present study.

hundred and seventy eight patients refracted in this study were aged over 60 . The average age of these patients is reflected in the frequency distribution of equivalent sphere refractive errors, shown in Fig. 2, which is statistically different to the distribution of refractive errors of other studies by Sorsby $e t$ $a l .{ }^{15}(\chi 2=512$ Degrees of Freedom D.F. $=8$, 
Table I. Reliability of Autorefractors and Conventional Refractive Techniques, Expressed in terms of test-retest Pearson product moment correlation (Rxy), the index of reliability (Rxy2), Standard Error of Measurement (SEM) and the Cumulative Difference Score for repeated autorefractor, retinoscopy and subjective measurements.

\begin{tabular}{|c|c|c|c|c|c|c|}
\hline \multirow{2}{*}{$\begin{array}{l}\text { Technique/Study } \\
\text { AUTOREFRACTORS }\end{array}$} & \multirow{2}{*}{$\begin{array}{l}\text { Refractive } \\
\text { Components }\end{array}$} & \multirow[t]{2}{*}{$R x y$} & \multirow[t]{2}{*}{$\begin{array}{c}R x y 2 \\
\text { (per cent) }\end{array}$} & \multirow[t]{2}{*}{$\begin{array}{l}S E M \\
(+/-)\end{array}$} & \multicolumn{2}{|c|}{$\begin{array}{c}\text { per cent of eyes lie } \\
\text { within }\end{array}$} \\
\hline & & & & & $\begin{array}{c}0.25 d s \\
d c / 5\end{array}$ & $\begin{array}{c}0.50 d s \\
d c / 10\end{array}$ \\
\hline Canon Ref-10 & Sphere & 0.97 & 0.95 & $0.86 \mathrm{ds}$ & 61 per cent & 81 per cent \\
\hline \multirow{2}{*}{ (Wood 1986) } & Cyl & 0.65 & 0.43 & $0.07 \mathrm{dc}$ & 56 per cent & 82 per cent \\
\hline & Axis & 0.96 & 0.93 & $15 \mathrm{deg}$ & 56 per cent & 78 per cent \\
\hline Dioptron V & Sphere & 0.76 & 0.58 & $4.31 \mathrm{ds}$ & 65 per cent & 72 per cent \\
\hline \multirow[t]{2}{*}{ (Wood 1986) } & Cyl & 0.22 & 0.04 & $2.40 \mathrm{dc}$ & 31 per cent & 53 per cent \\
\hline & Axis & 0.83 & 0.70 & $34 \mathrm{deg}$ & 65 per cent & 75 per cent \\
\hline Nidek 3300 & Sphere & 0.65 & 0.43 & $3.82 \mathrm{ds}$ & 74 per cent & 85 per cent \\
\hline \multirow[t]{2}{*}{ (Wood 1986) } & Cyl & 0.76 & 0.58 & $0.97 \mathrm{dc}$ & 61 per cent & 75 per cent \\
\hline & Axis & 0.98 & 0.96 & 11 degs & 55 per cent & 75 per cent \\
\hline Nikon 2000 & Sphere & 0.87 & 0.75 & $2.55 \mathrm{ds}$ & 60 per cent & 72 per cent \\
\hline \multirow[t]{2}{*}{ (Wood 1986) } & Cylinder & 0.81 & 0.66 & $0.78 \mathrm{dc}$ & 70 per cent & 81 per cent \\
\hline & Axis & 0.96 & 0.94 & $16 \mathrm{deg}$ & 39 per cent & 62 per cent \\
\hline \multicolumn{7}{|l|}{ RETINOSCOPY } \\
\hline Safir et al. $1970^{20}$ & Cylinder & & & & & 77 per cent \\
\hline \multicolumn{7}{|c|}{ Bizell et al. $1974^{11}$} \\
\hline \multicolumn{7}{|c|}{ SUBJECTIVE REFRACTION } \\
\hline \multirow{4}{*}{$\begin{array}{l}\text { French \& Jennings } \\
(1974)^{14}\end{array}$} & & & & & & \\
\hline & Sphere & 0.98 & 0.96 & $0.25 \mathrm{ds}$ & 68 per cent & \\
\hline & Cyl & 0.60 & 0.36 & $0.17 \mathrm{dc}$ & 85 per cent & \\
\hline & Axis & 0.91 & 0.82 & 18deg. & - & \\
\hline \multirow[t]{3}{*}{ Perrigin et al. $(1984)^{6}$} & Sphere & \multirow{3}{*}{\multicolumn{2}{|c|}{ Not Available }} & & 91 per cent & 100 per cent \\
\hline & Cyl & & & & 93 per cent & 99 per cent \\
\hline & Axis & & & & 71 per cent & 88 per cent \\
\hline
\end{tabular}

The above table is arranged so that a direct comparison between the reliability data of this study can be compared with other studies.

significance level $=0.01$ ) and Stenstrom ${ }^{16}$ $(\chi 2=159 \quad$ D.F. $=8, \quad$ significance level $=0.01)$.

\section{Reliability of Autorefractors}

The reliability statistics of the Canon Auto Ref 10, The Dioptron V the Nidek 3300 and Nikon 2000 autorefractors are shown in summary in Table I. These results have been produced from two autorefraction measurements of each instrument taken on two separate occasions. The reliability statistics of this study were generated from the Pearson Product Moment Coefficient, the Standard error of Measurement and the frequency dioptre difference statistics.

\section{The Correlation Coefficient of Reliability}

This describes how close the linear relationship is between the repeated spherical power and cylindrical power and axis measurements.
Complete agreement between these measurements plotted graphically would produce a straight line at 45 degrees to the ordinate and the abscissa. Both the slope and the Pearson Product Moment Coefficient will be 1. Squaring the Pearson Product Moment Coefficient yields the index of reliability or the percentage agreement between the repeated measurements.

The reliability correlation coefficients of this study for spherical components vary from 0.87 to 0.95 . The indices of reliability of this study indicate there is a 43 per cent to 95 per cent agreement between the repeated spherical component autorefraction measurements. These reliability statistics are therefore lower than the reliability statistics of other studies which show there is at least a 90 per cent agreement between the first and second autorefraction result. A smaller decrease in the reliability of the cylinder power and axis com- 
Table II. Validity of Autorefractors. Expressed in terms of test-retest Pearson product moment correlation (Rxy) and the index of Validity (Rxy2) and the magnitude of differences between autorefractor and conventional refraction measurements.

\begin{tabular}{lllcccc}
\hline $\begin{array}{l}\text { Autorefractorl } \\
\text { Study }\end{array}$ & $\begin{array}{l}\text { Refractive } \\
\text { Component }\end{array}$ & Rxy & Rxy2 & \multicolumn{3}{c}{ Mag of Differences } \\
& & & & $\begin{array}{c}>0.25 d \\
5 \text { deg } \\
\text { (per cent) }\end{array}$ & $\begin{array}{c}\text { (0.50d } \\
\text { (per cent) }\end{array}$ & $\begin{array}{c}<1.00 d \\
\text { (per deg } \\
\text { (pent) }\end{array}$ \\
\hline Canon Ref 10 & Spherical & 0.916 & 0.840 & 40 & 61 & 80 \\
(Wood 1986) & Cylinder & 0.692 & 0.479 & 43 & 74 & 85 \\
& Axis & 0.719 & 0.517 & 63 & 67 & 74 \\
Dioptron V & Spherical & 0.455 & 0.207 & 35 & 41 & 61 \\
(Wood 1986) & Cylinder & 0.450 & 0.202 & 30 & 51 & 66 \\
& Axis & Not Available & 66 & 70 & 76 \\
Humprehy 510 & Sphere & 0.552 & 0.271 & 46 & 55 & 75 \\
(Wood 1986) & Cylinder & 0.632 & 0.399 & 49 & 63 & 82 \\
& Axis & 0.462 & 0.215 & 74 & 80 & 84 \\
Nidek 3300 & Sphere & 0.947 & 0.897 & 52 & 72 & 88 \\
(Wood 1986) & Cylinder & 0.689 & 0.475 & 50 & 66 & 80 \\
& Axis & 0.781 & 0.611 & 64 & 66 & 74 \\
Nikon 2000 & Sphere & 0.954 & 0.911 & 43 & 66 & 83 \\
(Wood 1986) & Cylinder & 0.808 & 0.650 & 42 & 71 & 85 \\
& Axis & 0.916 & 0.839 & 68 & 70 & 86 \\
\hline
\end{tabular}

ponents is noted in this study when the correlation and index of reliability coefficients are compared with those of earlier studies.

\section{Cumulative Frequency Statistics}

An alternative and perhaps more familiar measure of reliability can be obtained from the cumulative frequency table of differences between repeated measurements. In Table I the cumulative frequency statistic describes the percentage difference of $0.25 / 0.50$ dioptre sphere/cylinder or 5/10 degree axis setting between first and second autorefraction measurements of all the eyes tested by each autorefractor. The step interval used to generate this statistic was 0.25 dioptre sphere, 0.25 dioptre cylinder and 5 degree axis setting.

The cumulative frequency table of the repeated difference scores confirm the poorer performance in this study in that only 40 per cent of the second sphere component measurement fall within plus $(+)$ or minus (-) 0.25 dioptre of the first measurement. The sixty ( 60 per cent) agreement between repeated cylinder power autorefraction measurements of plus $(+)$ or minus $(-) 0.25$ dioptre cylinder and the seventy five ( 75 per cent) agreement between repeated cylinder axis autorefraction measurements of plus $(+)$ or minus (-) 5 degrees, shows that the reliability of the autorefractors reviewed in this study are lower than those of other recent studies. ${ }^{1,13,17}$ Some autorefractors used in this study produce better results than those gained by repeated retinoscopy ${ }^{11,17}$ or repeated subjective results. Perrigin ${ }^{17}$ has pointed out that the high repeatability of some of these studies is greater than would be expected in optometric practice, as their subjects were to some extent trained observers.

\section{Standard Error of Measurement}

While the frequency analysis of differences is useful to the clinician it does not give an overall idea of the dispersion of the data. The standard deviation of repeated autorefractor or conventional refractive measurements and its derivative the standard error of measurement does give an overall idea of the dispersion of the data, assuming the distribution of the data is normal.

Further evidence of the decrease in the reliability of the four autorefractors used in this part of the study is reflected in the size of the standard error of the measurement (SEM) statistic. This is much larger for each refractive component than those of previous studies of repeated autorefractor measurements ${ }^{1,13,3}$ retinoscopy measurements ${ }^{18}$ and repeated subjective measurements. ${ }^{19,14}$ 


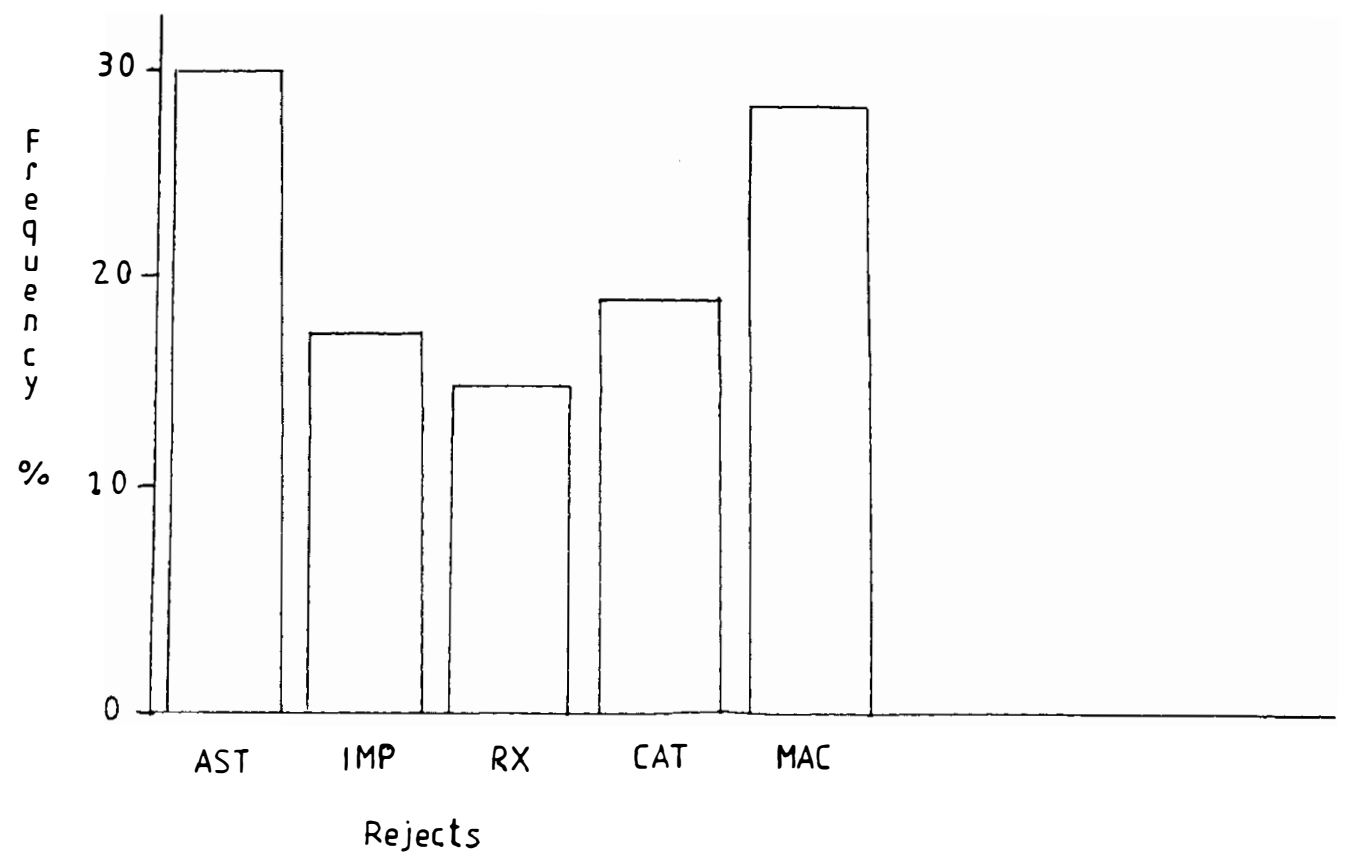

Fig. 3. Distribution of rejected autorefractor measurements for astigmatism (AST) greater than 2DC, implants $(I M P)$, spherical ametropia $(R X)$ greater than $+/-10 D S$, media opacities $(C A T)$ and macular pathology $(M A C)$.

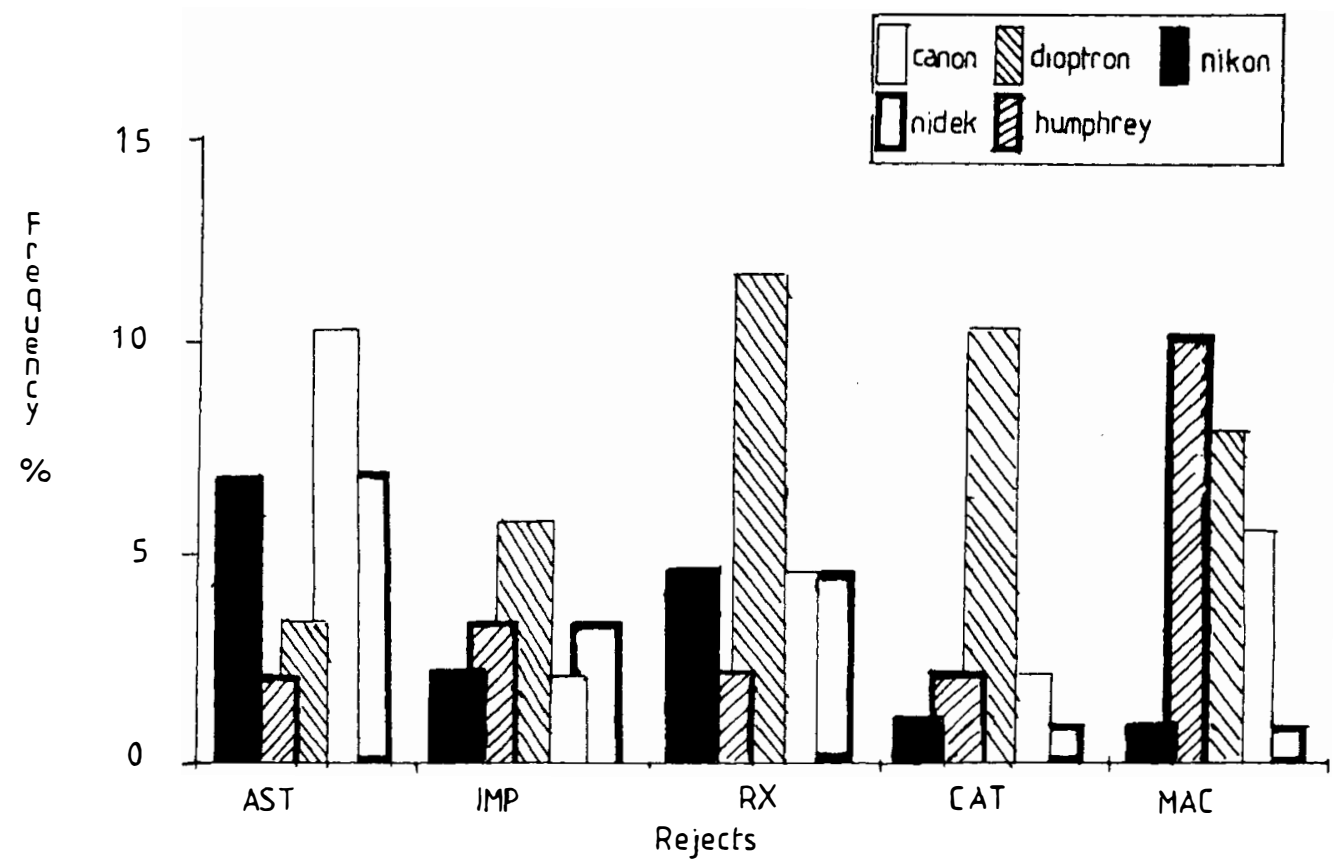

Fig. 4. Distribution of rejected measurements for each autorefractor:- Nidek 3300 (nidek), Dioptron V (dioptron), Nikon 2000 (nikon), Canon Ref 10 (canon) and the Humphrey 510 (hump). 


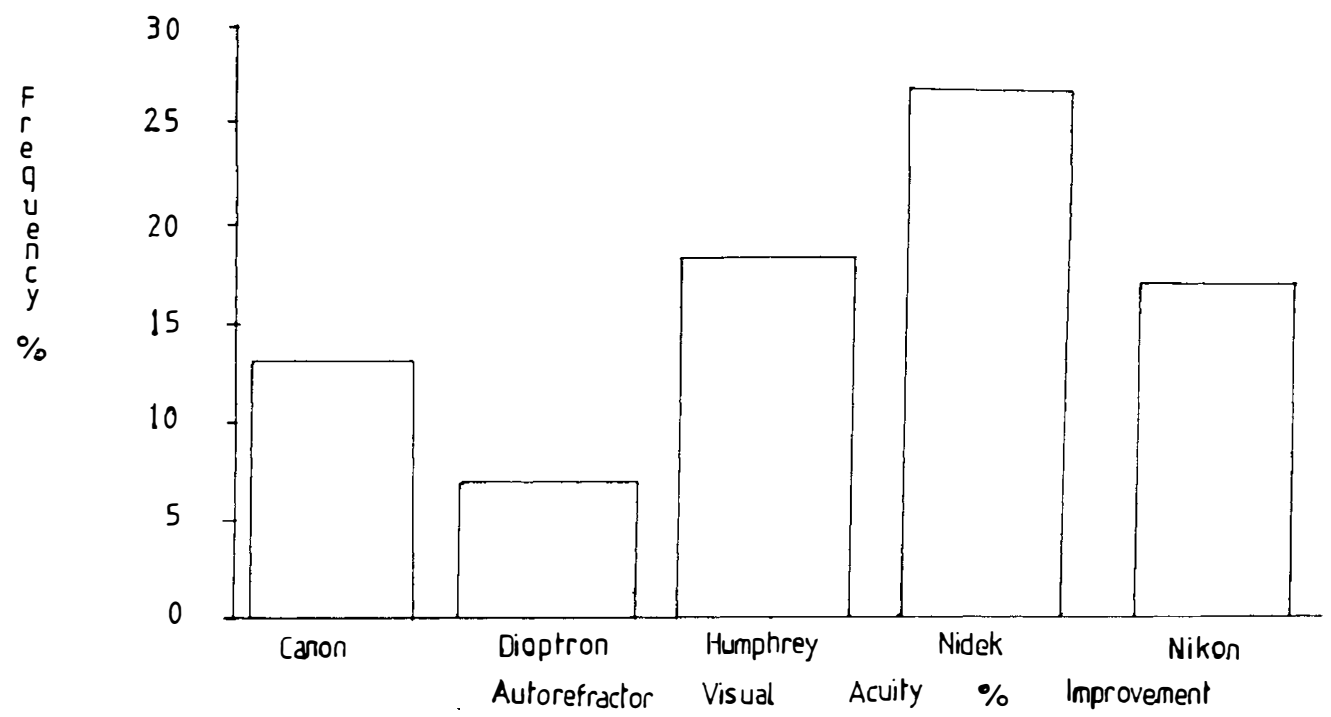

Fig. 5. Distribution of the percentage improved Snellen visual acuity of the autorefraction measurement by at least one line of 6 metre Snellen chart above that of the visual acuity obtained by conventional refraction methods.

\section{Validity of Autorefraction}

To determine the validity of autorefractors, blind autorefractor measurements are usually compared with the results obtained by conventional retinoscopy and subjective techniques using the Pearson product moment correlation coefficient and the magnitude of differences statistics (Table II).

\section{Discussion}

The present study shows a lower agreement of between 10 per cent to 20 per cent between the sphere and cylinder power components of infrared refractors and conventional refraction measurements than those of previous validity studies. There is in this study an even lower level of agreement of 30 per cent between cylinder axes measurements of the autorefractors and conventional refractive techniques. This may be because of the decrease in sensitivity in both conventional and autorefraction axis detection techniques for cylinder powers less than 1.00 dioptre cylinder. Therefore some studies of autorefractors quote the per cent agreement between autorefractors and conventional techniques with cylinders above $1.00 \mathrm{DC}$. The refractive data found by autorefractors are likely to need some subjective refinement. It is difficult to ignore the reliability studies of the final conventional refractive findings described above because these show that all conventional refraction techniques are themselves subject to error!

No matter how repeatable or valid the measurement of the autorefractor appears to be on those results, if the autorefractor rejects too many eyes then its value decreases. In this study, 25 per cent ( 87 out of the 378 ) of the autorefractor measurements were unobtainable or rejected where a conventional refraction measurement was possible. This figure is lower than the overall rejection figure of 60 per cent found by Kempster ${ }^{2}$ and the 40 per cent by Rassow and Wesemann. ${ }^{3}$

Figure 3 shows the overall percentage rejects broken down in terms of each of five factors:
(a) High Refractive Error ( $>10$ dioptres of ametropia
(b) Astigmatism greater than $2.00 \mathrm{DC}$
(c) Intraocular Implants (IOLS)
(d) Media Opacities
(e) Macular Pathology

as each of these factors will decrease the signal to noise ratio of each instrument. For instance forward scattering of the measuring infrared beam of an autorefractor through increased 
reflections from IOLS or media changes will increase noise ${ }^{21}$ whereas macular pathology changes the plane of polarisation of the measuring infrared beam. As illustrated by the early study of Polse and $\mathrm{Kerr}^{4}$ astigmatism greater than $2 \mathrm{DC}$ is likely to lower the signal to the autorefractor detector/servomechanisms. Similarly spherical ametropia greater than 10 dioptres is likely to lower the signal. From this figure it appears that macular changes and astigmatism greater than 2DC appear to be the principal cause of rejected readings. Figure 4 shows a further breakdown of the percentage rejects for each autorefractor for each of these five factors. This breakdown analysis seems to indicate that the autorefractors such as the Dioptron V, the Humphrey Autorefractor and Canon Autorefractor which, because they are based on image analysis methods of measurement, are affected by macular lesions. Those autoreactors based on retinoscopy (Nikon) or Coincidence/Schiener Disc methods of measurements are more likely to be affected by high levels of ametropia.

\section{Visual Acuity improvement by Autorefraction}

As a counterbalance to the rejection rate, it is evident that about $15 \%$ of the autorefractor measurements produced a higher visual acuity than that of conventional refracting techniques. Figure 5 shows the percentage of decimal visual acuity which were higher from the autorefractor than conventional refraction.

Despite the lower performance, autorefractors can be used by a technician as a preliminary refractive check to save time. Autorefraction should therefore be seen as a complement to subjective refraction.

\footnotetext{
References

${ }^{1}$ French CN, and Wood ICJ: The Dioptron in practice. Optician 1981, 181: 18-30.

${ }^{2}$ Kempster J: An evaluation of the Dioptron in Hospital Practice. Optician 1975, 160: 17-8.

${ }^{3}$ Rassow B and Wesemann W: Automatic infrared
}

refractors. Ophthalmology 1984, 91: Instr Book Suppl 10-26.

${ }^{4}$ Polse KA and Kerr KE: An automatic objective optometer. Arch Ophthalmology 1975, 93: 62730.

${ }^{5}$ Berman M, Nelson P, Caden B: Objective Refraction: Comparison of Retinoscopy and Automated Techniques. Am J Optom Physiol Opt 1984, 61: 204-9.

${ }^{6}$ Perrigin D, Grosvenor T, Reis A, Perrigin J: Comparison of Dioptron Nova Refractive data with Conventional refractive data. Am J Optom Physiol Opt 1984, 61: 496.

${ }^{7}$ Collins G: The Electronic Refractionometer. $\mathrm{Br} \mathrm{J}$ Physiol Optics 1937, 11: 30-42.

8 Wood ICJ: A comparative study of Autorefractors. Ophthal Optn 1982, 22: 221-5.

${ }^{9}$ Charman WN: Editorial: Infra-red Refractorshere to stay?. Ophthal Physiol Optics 1985, 5: 237-9.

${ }^{10}$ Dyson C: A clinical study of the autorefractor, an automatic refracting device. Can J Ophthalmol 1977, 96: 993-6.

${ }^{11}$ Bizzel FW, Hendricks JC, Goldberg F, Patel M, Robins GF: Clinical evaluation of an infra-red refracting instrument. Arch Ophthalmology 1974, 92: 103-8.

12 Wong EK, Patella V, Pratt M, Myers S, Gaster R, Irving HL: Clinical evaluation of the Humphrey Automatic Refractor. Arch Ophthalmology 1984, 102: 870-5.

${ }^{13}$ Wood ICJ, Papas E, Burghardt D, Hardwick G: A clinical evaluation of Nidek autorefractor. Ophthal Physiol Opt 1984, 4: 169-78.

${ }^{14}$ French $\mathrm{CN}$ and Jennings JAM: Errors in subjective refraction-an exploratory study. Ophthal Optn 1974, 14: 797-806.

${ }^{15}$ Sorsby A, Sheridan M, Leary GA: Vision, visual acuity and ocular refraction in young men. $\mathrm{Br}$ Med J 1960 1: 1394-8.

${ }^{16}$ Stenstrom S: Undersuchungen uber die Variation and Kovariation der optiscen Elemente des menschlichen Auges. Acta Ophthal, supplement 26. (English translation by Woolf D (1948). Am J Optom 1946, 25: 218-32.

${ }^{17}$ Perrigin J, Perrigin D, Grosvenor T: A comparison of clinical refractive data obtained by three examiners. Am J Optom Physiol Opt 1982, 59: 515-19.

${ }^{18}$ Hirsch MJ: Variability of retinoscopy measurements when applied to large groups of children under visual screening conditions. Am J Optom 1956, 32: $410-16$.

19 Jennings JAM and Charman WNC: A comparison of errors in some methods of subjective refraction. Ophthal Optn 1973, 6: 8-18.

${ }^{20}$ Safir A, Hyams L, Philpot J, Jaegerman LS: Studies in refraction 1 . The precision of retinoscopy. Archs Ophthalmol 1970, 84: 49-61.

${ }^{21}$ Charman N: A pioneering instrument. The Collins electronic refractionometer. Ophthal Optn 1976, 16: $345-8$. 